\title{
High Order Accurate Numerical Simulation of Vortex-Induced Vibrations of a Cooled Circular Cylinder Case using Solution Dependent Weighted Least Square Gradient Calculations
}

\author{
Chandrakant Sonawane ${ }^{1 *}$, Priyambada Praharaj ${ }^{1}$, Anand Pandey ${ }^{1}$, and Atul Kulkarni ${ }^{2}$ \\ ${ }^{1}$ Symbiosis Institute of Technology, Symbiosis International (Deemed University), Pune, India \\ ${ }^{2}$ Symbiosis Centre for Nano-Science and Nano-Technology, Symbiosis International (Deemed University), Pune, India
}

\begin{abstract}
In this paper, the fluid-structure interaction problem: vortex-induced vibration of a cooled circular cylinder involving thermal buoyancy is numerically investigated. The elastically mounted cylinder having a temperature lower than the flowing fluid is modelled using mass-spring-damper hence allowed to vibrate in the transverse direction to the flow direction. The gravity is acting opposite to the flow direction. In-house fluid-structure interaction solver is developed based on Harten Lax and van Leer with contact for artificial compressibility Riemann solver. The arbitrarily Lagrangian-Eulerian formulation is employed here, and the mesh is dynamically moved using radial basis function-based interpolation. The solutiondependent weighted least squares based gradient calculations are developed to achieve higher-order accuracy over unstructured meshes. The laminar incompressible flow at Reynolds number, $\operatorname{Re}=200$, and Prandtl number, $\operatorname{Pr}=0.71$, is simulated for the mass ratio of 1 and reduced damping coefficient of 0.001 . The flow is investigated for Richardson number $(-1,0)$ and over a wide range of natural frequencies of the cylinder. The heat transfer characteristics from a cylinder are captured and compared with the existing literature results. From the study, it can be observed that in the presence of the thermal boundary layer, the oscillation of the cylinder increases to its maximum amplitude, particularly for values of natural frequencies $(0.063-0.3)$.
\end{abstract}

\section{Introduction}

In engineering applications, flow over bluff bodies like circular, square, etc., are found almost in all applications. To mention few examples: flow over heat exchanger tubes, tubes in power plants, subsea cables for electronic communication and power transmission, submarine oil pipelines, offshore risers, etc. These structures experience the vibration mainly caused due to the surrounding fluid flow hence known as flowinduced vibration (FIV). When periodic lift and drag forces are generated over the cylinder due to separation and vortex shedding leading to structural vibrations are known explicitly as vortex-induced vibrations (VIV). This fluid-structure interaction (FSI) may result in serious engineering problems such as fatigue or fretting failure of the offshore riser and subsea pipelines, vibrations of a tube of the heat exchanger, vibration in structures such as a chimney, high rise building, etc. It has also been found that FIVs influence the convection heat transfer process, where the boundary layer separation, vortex shedding, and wake pattern are affected due to the FIV's. Hence, the prediction of fluid forces and heat transfer characteristics of a cylindrical structure and providing a reliable solution either to suppress or to utilize these vibrations have great importance.
VIV of a circular cylinder has been extensively studied over the last few decades, but the physics behind this complex FSI problem is still elusive and remains the core of the FIV modelling. A detailed review of FIV can be found in [1 - 3]. Recently, many researchers have investigated the FIV of circular/cylindrical structures involving heat transfer. Pottebaum and Gharib [4] experimentally investigated the heat transfer characteristics of a circular cylinder due to the transverse oscillations in cross-flow. They observe that the heat transfer enhancement is dependent on cylinder transverse velocity, the cylinder wake mode, and the synchronization with harmonics of the natural shedding frequency. Fu and Tong [5] to investigated the flow structures and heat transfer characteristics of a heated transversely oscillating cylinder in a cross-flow and found that the heat transfer is increased remarkably, particularly in the lock-in regime. Zhang et al. [6] numerically investigated the effect of Reynolds number at various temperature boundary conditions using the immersed-boundary method over a forced oscillating cylinder involving heat transfer.

Su et al. [7] used CFX-Ansys software to investigate the FIV and heat transfer of an elastically supported circular cylinder. They observed that cylinder vibration amplitude in the transverse direction is much larger than that of the streamwise direction. They also observed that

* Corresponding author: $\underline{\text { crsonawane@gmail.com }}$ 
heat transfer is not significant when the flow velocity is comparable to the cylinder oscillation velocity. Zhou et al. [8] used the discrete vortex method to study one and two degrees of freedom FIV of an elastically mounted circular cylinder. They observed a qualitative agreement between the simulated results obtained from the two degrees of freedom simulation with one degree of freedom. They obtained the maximum amplitude of cylinder vibration when the natural frequency of the fluid-structure system matches with cylinder oscillation frequency. Baratchi et al. [9] investigated the FIV of a heated elastically supported cylinder using the moving overset grids method. They studied the force coefficients, the amplitude of oscillations, vortex shedding pattern, and Nusselt number at $\mathrm{Re}=200$ and $\operatorname{Pr}=0.7$.

Wang et al. [10] investigated the forced convection heat transfer characteristics of a heated and elastically supported circular cylinder. They observed that the Nusselt number increases significantly in the lock-in region with increased cylinder vibrating amplitude. Wan and Patnaik [11] investigated the mixed convection heat transfer from elastically supported and heated circular cylinder under transverse VIV. They observed that the VIV of a cylinder could be entirely suppressed for critical values of Richardson number. Izadpanah et al. [12] also investigated the forced convection heat transfer characteristics of a heated and elastically supported circular cylinder under transverse VIV. They observed that heat transfer is significantly varied due to reduced velocity and damping ratio.

Chatterjee [13] has investigated the effect of thermal buoyancy on a stationary cylinder at a low Reynolds number. Sharma and Eswaran [14] also investigated the effect of buoyancy on the flow structure and heat transfer characteristics of an isolated square cylinder. Singh and Chandar [15] investigated the thermal effects on the vortex shedding produced by an unsteady flow over a circular cylinder at a Reynolds number of 150 . The flow direction and thermal buoyancy force were acting perpendicular to each other. To suppress the vortex shedding, they divided the cylinder into four quadrants and heated and cooled the different quadrants simultaneously. They also concluded that only heating the cylinder surface cannot suppress the vortex shedding completely.

Recently, Garg et al. [16] investigated the VIV of an elastically mounted circular cylinder in the presence of thermal buoyancy. The thermal buoyancy was induced by two parallel plates (bottom plate heated, and top plate was cooled). They studied the effect of Richardson number, Prandtl number, mass ratio, and reduced velocity on the cylinder vibrations, oscillation frequency, lift forces, and wake structure. They observe that the thermal buoyancy affect the cylinder vibrations are increases significantly for a wide range of reduced velocities. Garg et al. [17] also investigated the effect of thermal buoyancy over a heated and elastically supported circular cylinder for cross-flow conditions. At higher Richardson numbers, they observed the galloping responses due to the transversely acting thermal buoyancy. Garg et al. [18] further studied the effect of thermal buoyancy on a VIV of a cooled cylinder. They observed that when the flow is aligned in the opposite direction of the thermal buoyancy, a wider lock-in region along with a larger cylinder vibrating amplitude appears.

From the above literature review, it can be seen that very few investigations are available on the VIV of a circular cylinder involving thermal buoyancy. Hence, in this paper, we developed an in-house fluid-structure interaction solver for simulating the VIV of a cooled cylinder (cylinder surface is kept at a lower temperature than fluid flow temperature) involving opposing thermal buoyancy. An accurate Harten Lax and van Leer with contact for artificial compressibility (HLLC-AC) Riemann solver [21 - 24] are developed for solving incompressible flows in artificial compressibility formulation. The Riemann solver is modified to incorporate arbitrarily Lagrangian-Eulerian (ALE) [25, 19 - 20] formulation to take care of moving mesh scenarios where radial basis function [26] based interpolation is used for dynamically moving the mesh. Higher-order accuracy is achieved using solutiondependent weighted least squares (SDWLS) [27] based gradient calculations over unstructured meshes

\section{Arbitrarily Lagrangian-Eulerian (ALE) Formulation}

The dimensionless form of the governing equations for the unsteady incompressible flows involving the thermal buoyancy effect can be written as

$$
\begin{aligned}
& \frac{\partial u_{i}}{\partial x_{i}}=0 \\
& \frac{\partial u_{j}}{\partial t}+\frac{\partial u_{j} u_{i}}{\partial x_{i}}=-\frac{\partial p}{\partial x_{j}}+\frac{1}{R e} \frac{\partial}{\partial x_{i}}\left(\frac{\partial u_{j}}{\partial x_{i}}\right)+\delta_{2 j} \frac{G r}{R e^{2}} T \\
& \frac{\partial T}{\partial t}+u_{i} \frac{\partial T}{\partial x_{i}}=\frac{1}{R e \cdot P r}\left(\frac{\partial^{2} T}{\partial x_{j}^{2}}\right)
\end{aligned}
$$

where $\mathrm{i}=1$ and 2 correspond to the $\mathrm{X}$ and $\mathrm{Y}$ direction; respectively, all symbols are defined in the nomenclature. Equation (1) - (3) is modified using artificial compressibility [28] methods with a dual-time stepping approach. To take care of moving boundaries, an ALE formulation [25] is employed. Hence, the modified integral form of the two-dimensional incompressible flow can be written as

$$
\begin{array}{r}
\iint_{\Omega} \frac{\partial \mathrm{W}}{\partial \tau} \mathrm{dxdy}+\mathrm{I}^{\mathrm{M}} \iint_{\Omega} \frac{\partial \mathrm{W}}{\partial \mathrm{t}} \mathrm{dxdy}+\Theta^{\mathrm{M}} \oint_{\mathrm{A}}\left[\left(\mathrm{E}^{\mathrm{c}}+\right.\right. \\
\left.\left.\mathrm{E}^{\mathrm{v}}\right) \mathrm{n}_{\mathrm{x}}+\left(\mathrm{F}^{\mathrm{c}}+\mathrm{F}^{\mathrm{v}}\right) \mathrm{n}_{\mathrm{y}}\right] \mathrm{dA}=\iint_{\Omega} \mathrm{S}_{0} \mathrm{dxdy} \\
W=\left\{\begin{array}{c}
p / \rho \\
u \\
v \\
T
\end{array}\right\} ; E^{c}=\left\{\begin{array}{c}
U \\
U u+{ }^{p} / \rho \\
U v \\
U T
\end{array}\right\} ; \\
G^{c}=\left\{\begin{array}{c}
V \\
u V \\
v V+p / \rho \\
V T
\end{array}\right\} ; E^{v}=\left\{\begin{array}{c}
0 \\
\sigma_{x x} \\
\sigma_{x y} \\
\frac{1}{R e \cdot P r} \frac{\partial T}{\partial x}
\end{array}\right\} ;
\end{array}
$$




$$
\begin{gathered}
G^{v}=\left\{\begin{array}{c}
0 \\
\sigma_{y x} \\
\sigma_{y y} \\
\frac{1}{R e . P r} \frac{\partial T}{\partial y}
\end{array}\right\} ; I^{M}=\left\{\begin{array}{cccc}
1 & 0 & 0 & 0 \\
0 & 1 & 0 & 0 \\
0 & 0 & 1 & 0 \\
0 & 0 & 0 & 1
\end{array}\right\} \\
\Theta^{M}=\left\{\begin{array}{llll}
\beta^{2} & 0 & 0 & 0 \\
0 & 1 & 0 & 0 \\
0 & 0 & 1 & 0 \\
0 & 0 & 0 & 1
\end{array}\right\} ; S_{0}=\left\{\begin{array}{c}
0 \\
f_{e, x} \\
f_{e, y} \\
0
\end{array}\right\}
\end{gathered}
$$

Note that equation (4) is in the modified form, and once steady state in pseudo time is obtained, i.e. $\left(\frac{\partial p}{\partial \tau}=\frac{\partial u}{\partial \tau}=\right.$ $\left.\frac{\partial \mathrm{v}}{\partial \tau}=\frac{\partial \mathrm{T}}{\partial \tau} \cong 0\right)$ it recovers its original form as equation (3) written in ALE form.

For simplicity, convective fluxes $\left(E^{c}\right.$ and $\left.G^{c}\right)$ of equation (4) can be split into stationary reference flux and ale flux part as,

$\iint_{\Omega} \frac{\partial \mathrm{W}}{\partial \tau} \mathrm{dxdy}+\mathrm{I}^{\mathrm{M}} \iint_{\Omega} \frac{\partial \mathrm{W}}{\partial \mathrm{t}} \mathrm{dxdy}+\Theta^{\mathrm{M}} \oint_{\mathrm{A}}\left[\left(\mathrm{E}_{\mathrm{St}}^{\mathrm{c}}+\right.\right.$

$\left.\left.\mathrm{E}^{\mathrm{v}}\right) \mathrm{n}_{\mathrm{x}}+\left(\mathrm{F}_{\mathrm{st}}^{\mathrm{c}}+\mathrm{F}^{\mathrm{v}}\right) \mathrm{n}_{\mathrm{y}}\right] \mathrm{dA}-\oint_{\mathrm{A}}\left[\left(\mathrm{E}_{\text {ale }}^{\mathrm{c}}\right) \mathrm{n}_{\mathrm{x}}+\right.$

$\left.\left(\mathrm{F}_{\text {ale }}^{\mathrm{c}}\right) \mathrm{n}_{\mathrm{y}}\right] \mathrm{dA}=\iint_{\Omega} \mathrm{S}_{0} \mathrm{dxdy}$

The convective fluxes in equation (6) can be evaluated using HLLC-AC [21-25, 30] Riemann solver developed for stationary boundary problems. The ale flux vector is nothing but the volumetric increment along the face and can be evaluated by considering the Geometric Conservations Law (GCL) [26]. The radial basis function-based interpolation [29] is used here for dynamic mesh movement. The viscous fluxes are evaluated by the Green-Gauss approach based central differencing method.

\subsection{Solution reconstruction - evaluation of interface values of primitive variables $\left(W_{\mathrm{L}}\right.$ and $\mathbf{W}_{\mathrm{R}}$ ):}

Figure 1 shows the typical stencil used for unstructured meshes. Utilizing the Taylor series of expansion, the left and right state values of a flow variable, $\mathrm{W}$ at the interface of cells $i$ and $j$ can be given as

$$
\begin{aligned}
W_{L}=\bar{W}_{i}+\left(r_{i n t}\right. & \left.-r_{i}\right)^{T} \nabla \bar{W}_{i} \\
& +\frac{1}{2}\left(r_{i n t}-r_{i}\right)^{T} H_{i}\left(r_{i n t}-r_{i}\right)+\cdots
\end{aligned}
$$

$W_{R}=\bar{W}_{j}+\left(r_{i n t}-r_{j}\right)^{T} \nabla \bar{W}_{j}+\frac{1}{2}\left(r_{i n t}-r_{j}\right)^{T} H_{j}\left(r_{i n t}-\right.$ $\left.r_{j}\right)+\cdots$

The quadratic order of accuracy can be obtained by keeping three terms on the right-hand side (RHS) of the equation (7). Five derivatives of each flow variable, $\frac{\partial \bar{W}}{\partial x}, \frac{\partial \bar{W}}{\partial y}, \frac{\partial^{2} \bar{W}}{\partial x^{2}}, \frac{\partial^{2} \bar{W}}{\partial y^{2}}, \frac{\partial^{2} \bar{W}}{\partial x \partial y}$ at the cell centre, need to be evaluated for two-dimensional simulation.

\subsection{Solution dependent weighted least square (SDWLS)}

Figure 1 (b) shows the typical stencil for vertex-based neighbours used for the current weighted least squares formulation. For any cell $\mathrm{i}$ which has $\mathrm{n}$ number of neighbouring cells $(j=1, \ldots, n)$, using Taylor's series expansion applied over these neighbouring cells, the solution variable at the cell centre $i$ can be given as

$$
\begin{gathered}
\bar{W}_{j=1}=\bar{W}_{i}+\frac{\partial \bar{W}}{\partial x} \Delta x_{1}+\frac{\partial \bar{W}}{\partial y} \Delta y_{1}+\frac{\partial^{2} \bar{W}}{\partial x^{2}} \frac{\Delta x_{1}^{2}}{2} \\
+\frac{\partial^{2} \bar{W}}{\partial y^{2}} \frac{\Delta y_{1}^{2}}{2}+\frac{\partial^{2} \bar{W}}{\partial x \partial y} \Delta x_{1} \Delta y_{1} \\
\bar{W}_{j=2}=\bar{W}_{i}+\frac{\partial \bar{W}}{\partial x} \Delta x_{2}+\frac{\partial \bar{W}}{\partial y} \Delta y_{2}+\frac{\partial^{2} \bar{W}}{\partial x^{2}} \frac{\Delta x_{2}^{2}}{2} \\
+\frac{\partial^{2} \bar{W}}{\partial y^{2}} \frac{\Delta y_{2}^{2}}{2}+\frac{\partial^{2} \bar{W}}{\partial x \partial y} \Delta x_{2} \Delta y_{2}
\end{gathered}
$$
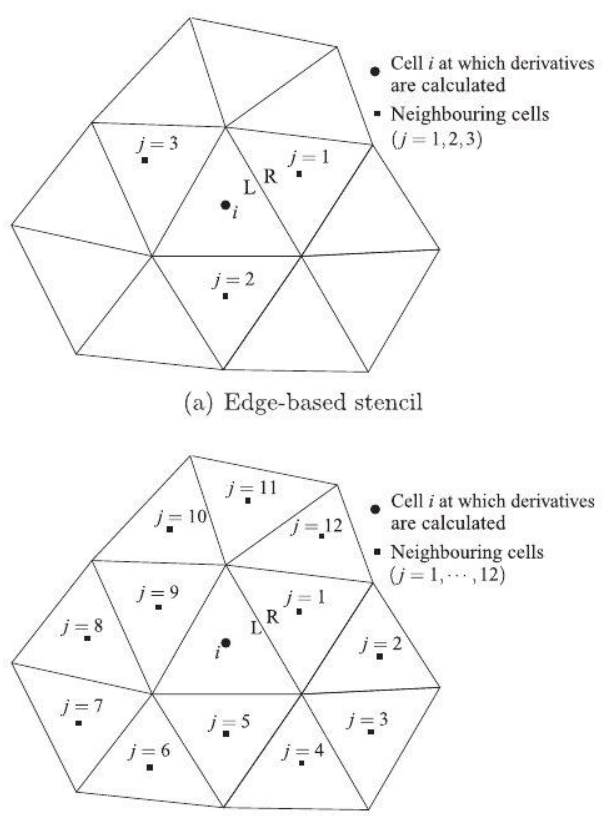

Fig. 1. Stencils for weighted least squares reconstruction (2D case).

Equation (8) can be summaries as an overdetermined system of equations as

$$
\Delta u=R d u
$$

where 


$$
\begin{aligned}
& \Delta u=\left\{\begin{array}{c}
\bar{W}_{j=1}-\bar{W}_{i} \\
\bar{W}_{j=2}-\bar{W}_{i} \\
\cdots \\
\cdots \\
\bar{W}_{j=n}-\bar{W}_{i}
\end{array}\right\} \\
& R=\left\{\begin{array}{ccccc}
\Delta x_{1} & \Delta y_{1} & \frac{\Delta x_{1}^{2}}{2} & \frac{\Delta y_{1}^{2}}{2} & \Delta x_{1} \Delta y_{1} \\
\Delta x_{2} & \Delta y_{2} & \frac{\Delta x_{2}^{2}}{2} & \frac{\Delta y_{2}^{2}}{2} & \Delta x_{2} \Delta y_{2} \\
& & \cdots: . & \\
\Delta x_{n} & \Delta y_{n} & \frac{\Delta x_{n}^{2}}{2} & \frac{\Delta y_{n}^{2}}{2} & \Delta x_{n} \Delta y_{n}
\end{array}\right\} \\
& d u=\left\{\frac{\partial \bar{W}}{\partial x} \frac{\partial \bar{W}}{\partial y} \frac{\partial^{2} \bar{W}}{\partial x^{2}} \frac{\partial^{2} \bar{W}}{\partial y^{2}} \frac{\partial^{2} \bar{W}}{\partial x \partial y}\right\}^{-1}
\end{aligned}
$$

The required spatial derivatives can be evaluated by solving the least square formulation with weights as

$\left(W_{t}^{1 / 2} R\right)^{T} W_{t}^{1 / 2} \Delta u=\left(W_{t}^{1 / 2} R\right)^{T}\left(W_{t}^{1 / 2} R\right) d u$

where $\mathrm{W}_{\mathrm{t}}=\operatorname{diag}\left[\mathrm{W}_{\mathrm{t}, 1}, \ldots, \mathrm{W}_{\mathrm{t}, \mathrm{n}}\right]$ is the diagonal weight matrix, which is dependent on the solution itself $[27,31]$ and is solved using the QR decomposition [32] algorithm.

\subsection{Structural modelling of flow-induced vibration}

As shown in figure 2, the mass-spring-damper system was used to model the elastically supported circular cylinder. The dynamic equation of this system can be written as

$\ddot{X}+4 \pi \xi f_{n} \dot{X}+4 \pi^{2} f_{n}^{2} X=\frac{C_{L}}{2 M^{*}}$

Here $X, \dot{X}$, and $\ddot{X}$ are the non-dimensional displacement, velocity, and acceleration of the cylinder, respectively. $M^{*}$ is a mass ratio, $M^{*}=m / \rho D^{2}$; the Strouhal number corresponding to the natural frequency $\left(f_{n}\right)$ of the cylinder can be defined as $S t_{n}=f_{n} D / U_{\infty}$; The reduced velocity, $U_{r}=1 / S t_{n}=U_{\infty} / D f_{n}$ and the damping ratio is $\xi=\frac{c^{*}}{c_{c}{ }^{*}}$.

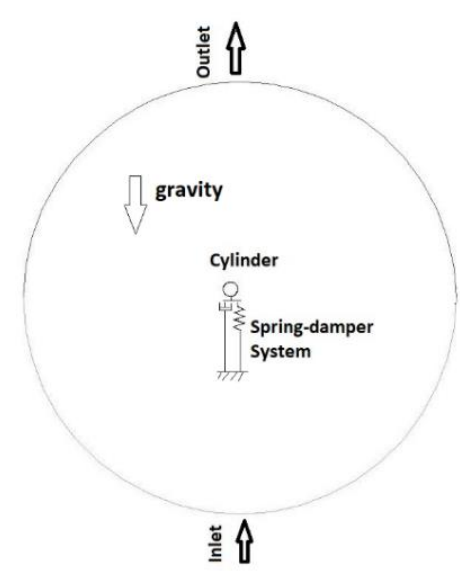

Fig. 2. The domain used for flow-induced vibration of an elastically supported circular cylinder with a mass-springdamper system.

\section{Result and Discussion}

Figure 2 shows the domain as well as boundary conditions used for VIV of a circular cylinder, where the outer boundary (inout) is located at 15 times diameter from the cylinder wall. The various boundary conditions are, at the inlet, vertical velocity (keeping $\mathrm{x}$ component of velocity as zero) is supplied corresponding to Reynolds number of 200, and pressure is interpolated from the interior domain. At the outlet, the atmospheric pressure is specified, and velocities are extrapolated from the interior domain. At the cylinder wall, the noslip boundary condition is maintained for the stationary cylinder, whereas for the VIV of a cylinder, the vibrating cylinder velocity is evaluated based on the structural dynamic equation and provided at the cylinder wall. Also, the cylinder wall is maintained at a constant temperature, which is at a temperature lower than that fluid flow temperature; hence the Richardson number is negative. The gravity is acting opposite to the flow direction.

Figure 3 shows the hybrid mesh used for the current study having 30410 mesh elements and 26216 nodes. The mesh is selected based on mesh convergence study (details are not presented here). The first quad mesh is located at a distance of 0.005 from the cylinder wall distance.

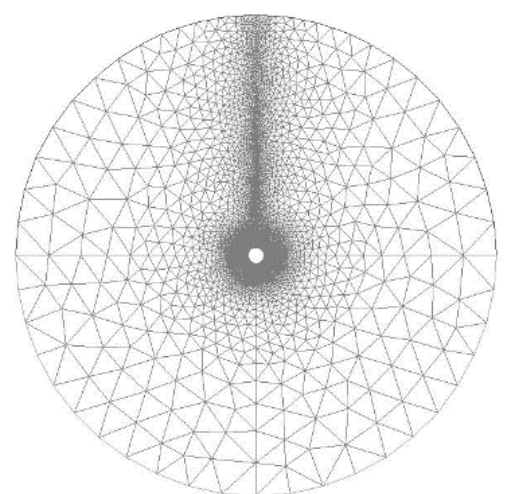

Fig. 3. Unstructured mesh used for vortex-induced vibration of a circular cylinder problem

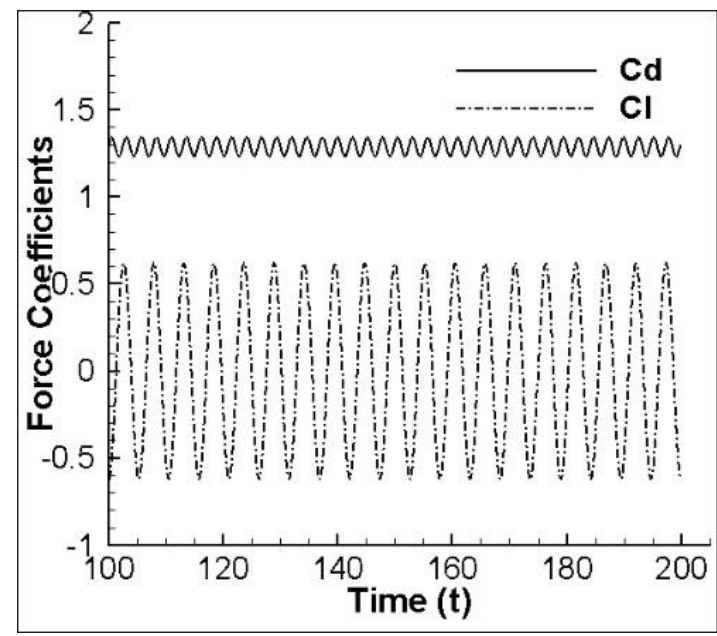

Fig. 4. Lift and drag coefficient for a stationary cylinder, $\mathrm{Re}=200$ and $\operatorname{Pr}=0.71$. 
Table 1: Comparison between Nusselt Number

\begin{tabular}{|c|c|c|}
\hline $\begin{array}{c}\text { Reynolds no } \\
\text { (Re) }\end{array}$ & \multicolumn{2}{|c|}{$\begin{array}{c}\text { Avg. Nusselt number } \\
\text { (Nu) }\end{array}$} \\
\hline & Baratchi et al. [9] & Present \\
\hline 200 & 7.11 & 7.22 \\
\hline
\end{tabular}

Initially, unsteady flow over a stationary cylinder at Reynolds number of 200 and Prandtl number of 0.71 is simulated. Figure 4 shows the lift and drag coefficient, whereas Table 1 shows the variation of Nusselt number for stationary cylinder compared with the numerical results of Baratchi et al. [9].

To investigate the VIV of the circular cylinder under the influence of thermal buoyancy, now the cylinder is allowed to vibrate in a transverse direction. The gravity is acting opposite to the flow direction. The cylinder is maintained at a lower temperature than the fluid temperature; hence the Richardson no, is negative. Firstly results for $\mathrm{Ri}=0$, i.e. in the absence of thermal buoyancy, are simulated.

Figure 5 shows the comparison of present results, cylinder amplitude achieved for a range of Strouhal number achieved due to the VIV of a circular cylinder without thermal buoyancy $(\mathrm{Ri}=0)$, with that of Baratchi et al. [9]. Figure 5 also shows the VIV characteristics with thermal buoyancy effect for $\mathrm{Ri}=-1$ condition. It can be observed that due to thermal buoyancy, the cylinder vibration increases significantly, particularly for the smaller strouhal number values. It can also be seen that the cylinder vibrates with a larger amplitude for a broader range of strouhal numbers

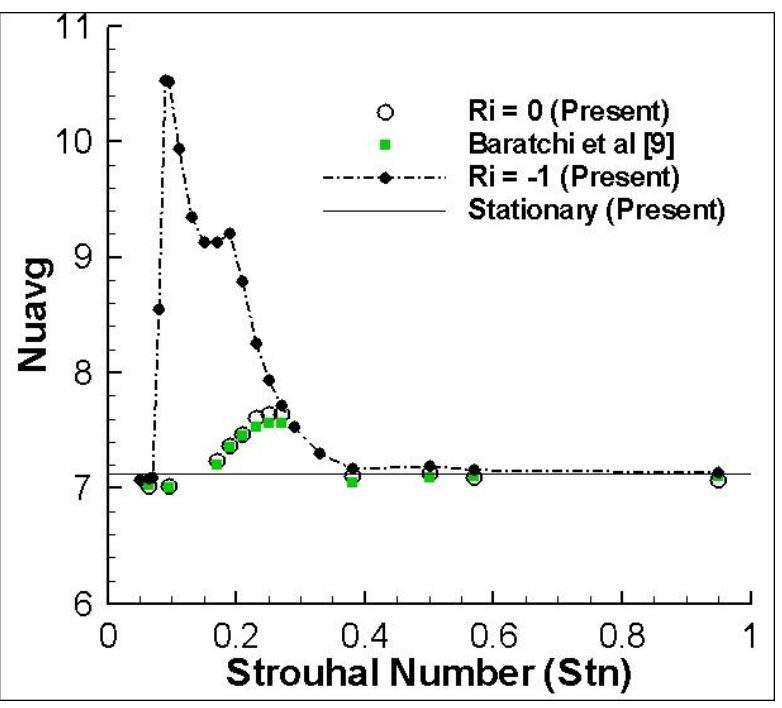

Fig. 5. Cylinder displacement obtained for VIV of a circular cylinder for $\mathrm{Re}=200$ and $\mathrm{Pr}=0.71$

\section{Conclusions}

The laminar incompressible flow at Reynolds number, $R e=200$, and Prandtl number, $\operatorname{Pr}=0.71$, is simulated for Richardson number $(-1,0)$ and over a wide range of natural frequencies of the cylinder. It can be seen that that in the presence of the thermal boundary layer, the oscillation of the cylinder increases to its maximum amplitude, particularly for values of natural frequencies $(0.063$ $-0.3)$

\section{Acknowledgements}

This work is funded and supported by the Science and Engineering Research Board (SERB) - Department of Science and Technology (DST), Government of India (ECR/2017/000476)

\section{References}

1. Sarpkaya T., A critical review of the intrinsic nature of vortex-induced vibrations, Journal of Fluids and Structures, 19(4), pp 389-447, 2004. https://doi.org/10.1016/j.jfluidstructs.2004.02.005

2. Williamson C. H. K. and Govardhan R., A brief review of recent results in vortex-induced vibrations, Journal of Wind Engineering and Industrial Aerodynamics, 96(6-7), pp 713-735, 2008. https://doi.org/10.1016/j.jweia.2007.06.019

3. Wang Js., Fan D. and Lin K., A review on flowinduced vibration of offshore circular cylinders, J Hydrodyn, 32, pp 415-440, 2020. https://doi.org/10.1007/s42241-020-0032-2

4. Pottebaum T., and Gharib M., Using Oscillations to Enhance Heat Transfer for a Circular Cylinder, Int. J. Heat Mass Transfer, 49 (17-18), pp. 3190-3210, 2006.

https://doi.org/10.1016/j.ijheatmasstransfer.2006.0 1.037

5. Fu W. S., and Tong B. H., Numerical Investigation of Heat Transfer From a Heated Oscillating Cylinder in a Cross Flow, Int. J. Heat Mass Transfer, 45, pp. 3033-3043, 2002. https://doi.org/10.1016/S0017-9310(02)00016-9

6. Zhang N., Zheng Z. C., and Eckels S., Study of Heat Transfer on the Surface of a Circular Cylinder in Flow Using an Immersed-Boundary Method, Int. J. Heat Fluid Flow, 29(6), pp. 1558-1566, 2008. https://doi.org/10.1016/j.ijheatfluidflow.2008.08.0 09

7. Su Y. C., Ge P. Q., and Yan K., Numerical Analysis on Heat Transfer and Flow-Induced Vibration of an Elastically Supported Cylinder, Mater. Sci. Forum, 697-698, pp. 585-589, 2011. https://doi.org/10.4028/www.scientific.net/MSF.6 97-698.585

8. Zhou C. Y., So R. M., and Lam, K., Vortex-Induced Vibrations of an Elastic Circular Cylinder, J. Fluids Struct., 13, pp. 165-189, 1999. https://doi.org/10.1006/jfls.1998.0195

9. Baratchi F, Saghafian M, and Baratchi B, Numerical investigation on lock-on condition and convective heat transfer from an elastically supported cylinder in a cross flow, J. Fluid Eng.-T. ASME 135:031103, 2013. https://doi.org/10.1115/1.4023192

10. Wang S. K., Hung T. C., Lin G. W., and Pei B. S., Numerical simulations for the phenomena of 
vortex-induced vibration and heat transfer of a circular cylinder, Numer. Heat Tr. A-Appl., 45, pp 719-36, 2014. https://doi.org/10.1080/10407780490424901

11. Wan H., and Patnaik S. S., Suppression of vortexinduced vibration of a circular cylinder using thermal effects, Phys. Fluids, 28:123603, 2016. https://doi.org/10.1063/1.4972178

12. Izadpanah E., Amini Y., and Ashouri A. A., comprehensive investigation of vortex induced vibration effects on the heat transfer from a circular cylinder, Int. J. Therm. Sci., 125 pp 405-18, 2018. https://doi.org/10.1016/j.ijthermalsci.2017.12.011

13. D. Chatterjee, Dual role of thermal buoyancy in controlling boundary layer separation around bluff obstacles, Int. Commun. Heat Mass Transfer, 56, pp 152-158, 2014. https://doi.org/10.1016/j.icheatmasstransfer.2014.0 6.012

14. A. Sharma and V. Eswaran, Effect of aiding and opposing buoyancy on the heat and fluid flow across a square cylinder at $\mathrm{Re}=100$, Numer. Heat Transfer, Part A 45, pp 601-624, 2004. https://doi.org/10.1080/10407780490277798

15. S. Singh and D. Chandar, Effects of thermal induced buoyancy forces on the vortex shedding of a circular cylinder, Int. Commun. Heat Mass Transfer, 76, pp 215-224, 2016. https://doi.org/10.1016/j.icheatmasstransfer.2016.0 5.012

16. H. Garg, A. K. Soti, and R. Bhardwaj, A sharp interface immersed boundary method for vortexinduced vibration in the presence of thermal buoyancy, Phys. Fluids, 30 (2), 023603, 2018. https://doi.org/10.1063/1.5008474

17. Garg H., Soti A. K., and Bhardwaj R., Vortexinduced vibration and galloping of a circular cylinder in presence of cross-flow thermal buoyancy. Phys. Fluids, 31, 113603, 2019. https://doi.org/10.1063/1.5122851

18. Garg H., Soti A. K., and Bhardwaj R., Vortexinduced vibration of a cooled circular cylinder, Phys. Fluids, 31, 083608, 2019. https://doi.org/10.1063/1.5112140

19. C. R. Sonawane, Y. B. More, Anand Kumar Pandey, "Numerical simulation of unsteady channel flow with a moving indentation using solution dependent weighted least squares based gradients calculations over unstructured mesh", Heat Transfer Engineering Journal, 2021, doi: https://doi.org/10.1080/01457632.2021.1874661

20. Sonawane Chandrakant, Hitesh Panchal \& Kishor Kumar Sadasivuni (2021) Numerical simulation of flow-through heat exchanger having helical flow passage using high order accurate solution dependent weighted least square based gradient calculations, Energy Sources, Part A: Recovery, Utilization, and Environmental Effects, DOI: 10.1080/15567036.2021.1900457
21. J C Mandal, C R Sonawane, A S Iyer and S J GosaviInamdar, Incompressible flow computations over moving boundary using a novel upwind method, Computers \& Fluids, 46 (1), pp 348-352, 2011. https://doi.org/10.1016/j.compfluid.2010.08.011

22. Mandal, J. C. and Sonawane, C. R., Simulation of flow inside differentially heated rotating cavity, International Journal of Numerical Methods for Heat \& Fluid Flow, Vol. 23 No. 1, pp. 23-54, 2013 https://doi.org/10.1108/09615531311289097

23. C R Sonawane and J C Mandal, Simulation of moderator flow and temperature inside calandria of CANDU reactor using artificial compressibility method, Journal of Heat Transfer Engineering, Vol 35, Issue 11/12, July/August 2014.https://doi.org/10.1080/01457632.2013.8768 02

24. C. R. Sonawane, Y. B. More and A. Pandey, Numerical Simulation of Unsteady Channel Flow with a Moving Indentation Using Solution Dependent Weighted Least Squares Based Gradients Calculations over Unstructured Mesh, Heat Transfer Engineering, 2021, DOI: 10.1080/01457632.2021.1874661

25. Cook J L, Hirt C W, Amsden A A, An arbitrary Lagrangian-Eulerian computing method for all flow speeds, Journal of Computational Physics, 14, Issue 3, pp. 227-253, March 1974. https://doi.org/10.1016/0021-9991(74)90051-5

26. H Bijl, A de Boer, M S van der Schoot, Mesh deformation based on radial basis function interpolation, Computers and Structures, 85 (1114), pp 784-795, 2007. https://doi.org/10.1016/j.compstruc.2007.01.013

27. Sonawane, C.R., Mandal, J.C. and Rao, S. High Resolution Incompressible Flow Computations over Unstructured Mesh using SDWLS Gradients. J. Inst. Eng. India Ser. C 100, pp 83-96, 2019. https://doi.org/10.1007/s40032-017-0390-x

28. A J Chorin, Numerical solution of Navier-Stokes equations, Math Comput, 22, 745-762, 1968. https://doi.org/10.2307/2004575

29. Lombard C K, Thomas P D, Geometric conservation law and its application to flow computations on moving grids. AIAA Journal, 17:1030-1037, 1979.

30. E. F. Toro, M. Spruce, W. Speares. Restoration of the contact surface in the HLL-Riemann solver. Shock Waves, 4, 25-34, 1994.

31. J. C. Mandal, S. P. Rao. High-resolution finite volume computations on unstructured grids using solution dependent weighted least squares gradients. Computers and Fluids, 44, 23-31, 2011.

32. C. F. V. Loan, G. H. Golub. Matrix computations, 3rd ed. The Johns Hopkins University Press, 1996. 